\title{
Epidemiological aspects of influenza in man and other animals
}

\author{
DEREK HOBSON \\ M.D., M.R.C.Path.
}

Department of Medical Microbiology, University of Liverpool, Liverpool

\begin{abstract}
Summary
The recurrent problem in human influenza is the pandemic increase in mortality and social dislocation which occurs every 10-15 years whenever a new subtype of type A virus emerges, with antigens which cannot be neutralized by antibodies induced in the population by infection or vaccination with any of the foregoing epidemic strains.

There is an urgent need to interpret these past antigenic shifts so that the constitution of the next pandemic strain can be predicted, allowing effective specific vaccines to be made in advance.

The pandemic viruses are unlikely to be direct mutants from one to another. Epidemiological evidence of their cyclic recurrence in mankind at 70 year intervals suggests the role of animal reservoirs of influenza viruses. Man may be infected directly, or after genetic recombination between an animal virus with novel antigens and a human strain with the necessary virulence for man.
\end{abstract}

THE Hong Kong influenza pandemic of 1968 is only the latest of a long series of abrupt changes in the incidence and severity of influenza. In 1957, 1947, $1930,1918,1900,1889$, and at intervals of $10-40$ years as far back as the 17th century (Francis, 1961) there are clear accounts of similar explosive pandemics, each followed by a gradual decrease in the size and frequency of outbreaks, and sometimes a gap of many years free from obvious influenza, until the next cycle began in a similar dramatic fashion.

Each successive pandemic from 1918 to 1968 is known to have resulted from the sudden emergence of one variant after another of influenza A virus. Each new subtype has shared the type-common RNP(S) antigen, but has differed so radically from its predecessors in one or both of the viral surface antigens, the haemagglutinin (HA) or neuraminidase $(\mathrm{N})$, that it successfully evaded all the immunity the population had acquired by previous epidemics or vaccination with any of the foregoing virus strains.

The relative importance of antibodies against the independently-coded $\mathrm{HA}$ and $\mathrm{N}$ proteins has been the subject of much recent debate (see Dowdle,
1972). The HA spikes on the virion act as an $\overrightarrow{\vec{\omega}}$ 'attachment organ', without which the particle cannot link on to, and subsequently infect, the suscep- 8 tible respiratory cell. HA-inhibiting (HI) antibody is thus clearly concerned with neutralizing virus $\overrightarrow{0}$ infectivity. The viral enzyme ( $\mathbf{N}$ antigen) is probably i not essential for the virus to initiate infection but aids the release of new virus progeny from infected respiratory cells by digesting linkages between the virus and cell-wall complexes containing bound neuraminic acid. Thus neuraminidase-inhibiting (NI) antibody does not prevent infection, but can limit the amount of virus shed from infected cells, and thus helps to control the rate of extension of the infection (Seto \& Rott, 1966; Jahiel \& Kilbourne 1966).

Whenever a new virus subtype has emerged, with new antigens against which the population has no prior immunity, the epidemiological picture has been remarkably similar. The new subtype appears quite suddenly, often early in the summer, and often in one geographic locality, e.g. Central China, and behaves as if it were a wholly new entrant to human disease, rather than the end stage of serial minor modifications of the preceding epidemic strains. The first introduction into densely-populated areas results in explosive outbreaks, with rapid case-tocase spread through one country after another in a remarkably linear fashion along established routes of transportation, progressing across the whole Northern Hemisphere in the first autumn and winter, but only spreading extensively in the Southern Hemisphere when their winter begins. Each hemisphere probably constitutes the reservoir from which the other is reinfected at yearly or 2-yearly intervals, until eventually such massive numbers have become immunized that the chain of virus transmission is broken. Influenza then becomes trivial and sporadic, until yet another type A virus with yet another antigenic variation appears, and can spread with impunity through the same populations to enjoy its own decade of epidemicity before being displaced in its turn by yet another virus. It is a remarkable feature of influenza that no two subtypes of type A virus have ever circulated con- 
currently in human populations, except at the point of changeover from minor to major epidemic behaviour. Once a new subtype has established itself, it dominates the whole world scene of influenza and the foregoing antigenic variant disappears from mankind, apparently forever.

By 1972 over $70 \%$ of the population in Britain and many other countries had acquired protective antibodies against $\mathrm{HK}$ virus, and if the past history of influenza is any guide to the future, a new contender of entirely different antigenic constitution will replace HK virus in the next few years, and usher in yet another pandemic period.

There is no certain way of forecasting what antigenic form this next virus will take, whether it will display entirely novel antigens or revert to one of the former constitutions; but it is predictable that once the new virus emerges, all immunity against $\mathrm{HK}$ virus will become irrelevant to the new situation and all stock-piles of $\mathrm{HK}$ vaccine become obsolete. It can take 6 months or more to make vaccine on a commercial scale against a new virus which has already begun its pandemic spread, but with our present lack of knowledge no prospective vaccine can be made before the new strain appears. In every past cycle of influenza the highest case-mortality, particularly in the young adult population, has occurred in the first major waves of infection; for example, over 10 million people died during the pandemic of 1918-19, the first epidemic of 1968-69 in the U.S.A. accounted for 58,000 deaths, and over 9000 deaths occurred in Britain within 3 weeks of the HK outbreak of 1969-70. There is thus an obvious need to learn enough about the long-term behaviour of influenza $A$ viruses to try to predict the next virus and make effective vaccines well in advance.

The general view in the U.S.A. until a few years ago was that all of the pandemic subtypes of influenza A shared all the possible HA antigens and merely changed their relative proportions according to the immunological barrier erected in the population. This view is now untenable for many reasons, and especially because the genetic apparatus of myxoviruses is too small to code for the large number of separate proteins each virus would need to contain. The conflicting British view of that time was that each pandemic subtype contained a single HA antigen, showing a progressive forward mutation from its immediate precursor under the selective pressure of population immunity. This also had to be discarded when it became obvious that the Asian (A2) strains differed from the foregoing A1 in $\mathrm{HA}$ and in $\mathrm{N}$ antigen and in other biological properties-far too massive an array of changes for one virus to be a point mutant of the other.

A more likely interpretation of influenzal history is that the A virus of $1918, \mathrm{~A} 0, \mathrm{~A} 1, \mathrm{~A} 2$, and $\mathrm{HK}$ viruses are, and always have existed as, fixed immutable subtypes, each in turn able to become pandemic only after the death of the last population cohort that had met it before and had preserved an immune memory of it. It is true that these subtypes cannot be made to mutate from one to the other in the laboratory, and strong indirect evidence in favour of this hypothesis, although it was originally developed to investigate earlier possibilities, is found in the long and careful studies of immunological archeology' from Ann Arbor (Davenport, Hennessy \& Francis, 1953). From these, it is clear that most people today are still manufacturing antibody they first learned to produce in specific response to the first influenza virus they encountered in their childhood. The over 50-year-olds are thus the only cohort with antibody against the 1918 virus, only those over 30 react with $\mathrm{A} 0$ virus, and the young children of 1960-72 show no antibody 'earlier' than anti-A2 and anti-HK, which they will probably continue to produce as evidence of their 'original antigenic sin' long after these viruses have disappeared from the scene. Mulder \& Masurel in Holland were probably the first to appreciate that it was logical to extend this 'doctrine of original antigenic sin' backwards, and that identification of the dominant or 'earliest' antibody present in the oldest age groups alive today would give strong indirect evidence of the subtype of virus which was pandemic in their own childhood, 70-80 years ago. They showed that people born about 1890 uniquely had antibody against A2 virus before the 'new' virus had reached Holland (Mulder \& Masurel, 1958) and that sera collected before 1957 from people born about 1900 had antibody active against the HK strain, which did not 'appear' until 1968 (Masurel, 1969). Thus, by a combination of direct virus isolation and serological detective work it seems likely that the biblical three score years and ten of human life encompasses a complete cycle of influenza viruses. Every observed pandemic from 1890-1947 can be assigned its own virus subtype, and what we have seen in 1957 and 1968 appears to be the start of another cycle of the same viruses appearing in the same order, with a distinct possibility that the next pandemic will be due to a second advent of a virus antigenically similar to the A 1918 strain, against which no people under 50 years of age have any immunological memory.

The credibility of this cyclic hypothesis would be considerably greater if there was any satisfactory explanation of where these subtypes of influenza $\mathbf{A}$ persist in the 50 years or more when they are certainly absent from mankind. The obvious possibility is that they circulate through animal reservoirs (Pereira, 1969), and it has long been known 
that the still common strain of Swine influenza is a type A virus which reacts uniquely with antibodies in the sera of people who experienced the 1918 pandemic. Similarly, viruses with antigens like those of human strains can be found in birds, and epizootics of influenza in horses have yielded type $A$ viruses with the HA resembling those of human A2-HK strains. Recently, viruses apparently identical with HK virus have appeared in epizootics of Swine influenza in Taiwan (Kundin, 1970). Thus, at a fairly superficial level the idea of direct and permanent animal reservoirs of influenza viruses, from which man can be subsequently infected, seems attractive. Further investigation, however, quickly exposes fallacies in the argument.

There is epidemiological evidence that Swine influenza outbreaks by 1918-style virus only began several years after human spread had occurred. Similarly, Taiwan Swine influenza began a year or more after the human HK pandemic. The Taiwan virus recovered from pigs has extremely poor infectivity for man, and despite many epizootics of $\mathrm{A} /$ Swine/15/31 influenza in pigs in the last 40 years there has been no proven spread of infection to pighandlers or the population at large, despite the almost universal lack of antibody in young people. But the most incontrovertible evidence against animal viruses being the direct source of human outbreaks is that, although they may cross-react with antibody against the HA antigen or the $\mathbf{N}$ antigen of human strains they seldom react with both, and differ in a number of biological properties quite apart from virulence.

The most recent hypothesis is based on a now historic finding that different influenza A viruses can undergo genetic recombination in doubly-infected cells to yield true-breeding progeny with genetic attributes derived from both parents (Burnet \& Lind, 1951). More recently, recombinant viruses have been bred with the HA of fowl plague virus plus the $\mathrm{N}$ antigen of $\mathrm{HK}$, or with the HA of $\mathrm{HK}$ plus the N of FPV (Tumova \& Pereira, 1965; Kilbourne et al., 1967), and the X31 recombinant, with both the $\mathbf{N}$ and $\mathrm{HA}$ antigens of $\mathrm{HK}$ crossed with the high growth capacity in eggs of laboratory-adapted A0/PR8 virus, is already widely used in the commercial manufacture of influenza vaccines.

The hypothesis for the 1970's is that animal strains of influenza A viruses might thus constitute a reservoir of genes able to code for antigens capable of evading the prevailing human immunological barriers; these animal viruses per se lack virulence for man and can only become successful invaders by recombination with human type $A$ strains which possess the right genes for human virulence but can make no further headway because their code for $\mathrm{HA}$ and $\mathrm{N}$ antigens is obsolescent. The right sort of double infection might happen only once in many million animal-to-man exchanges of virus, but perhaps once in a decade in some distant tundra an influenzal Mongolian sharing his yurt with an ailing member of his herd is all that is needed to explain our recurrent pandemics. It has already been shown by Webster \& Laver (1972) that new recombinants can arise in vivo when groups of pigs or turkeys deliberately infected with different strains of influenza virus are herded together.

This new concept, as it now stands, may well join the discarded hypotheses of the past in a few years. However, its practical value lies in its stimulus to prove that influenza $A$ antigens are immutable in nature and finite in number, and to encourage control laboratories to assemble a 'library' of every known $\mathrm{N}$ and $\mathrm{HA}$ antigen in the animal kingdom in every possible genetic combination, ready to go straight into vaccine production as soon as, or even before, the inevitable successor to HK virus appears.

\section{References}

BURNET, F.M. \& LiND, P.E. (1951) A genetic approach to variation in influenza viruses. IV. Recombination of $D$ characters between the influenza virus A strain NWS and strains of different serological subtypes. Journal of $\frac{\bar{C}}{\omega}$ General Microbiology, 5, 67.

DaVenPORT, F.M., HeNNeSSY, A.V. \& Francis, T. JR (1953 Epidemiologic and immunologic significance of age distrp bution of antibody to antigenic variants of influenza virus Journal of Experimental Medicine, 98, 641.

DowDLE, W.R. (1972) Influenza anti-neuraminidase: the second-best antibody. New England Journal of Medicine, 286, 1360.

Francis, T. JR (1961) Influenza in perspective. American Review of Respiratory Disease, 83, 98.

JAHIEL, R.I. \& KILBOURNE, E.D. (1966) Reduction in plaque size and reduction in plaque numbers as different indices of influenza virus-antibody reaction. Journal of Bacteriology, 92, 1521.

KILbourNe, E.D., Lief, F.S., Schulman, J.L., JAHIEl, R.I. \& LAVER, W.G. (1967) Antigenic hybrids of influenza viruses and their implications. Perspectives inVirology, 5, 87.

KundIN, W.D. (1970) Hong Kong A2 influenza virus infection among Swine during a human epidemic in Taiwan. Nature, 228, 857.

MASUREL, N. (1969) Serological characteristics of a 'new' serotype of influenza A virus: the Hong Kong strain. Bulletin of the World Health Organization, 41, 461.

Mulder, J. \& Masurel, N. (1958) Pre-epidemic antibody against 1957 strain of Asiatic influenza in serum of old people living in the Netherlands. Lancet, i, 810.

Pereira, H.G. (1969) Influenza: antigenic spectrum. In: Progress in Medical Virology, vol. 11, p. 46. Karger, Basle.

Seto, J.T. \& RotT, R. (1966) Functional significance of sialidase during influenza virus replication. Virology, 30, 731.

Tumova, B. \& Pereira, H.G. (1965) Genetic interaction between influenza $A$ viruses of human and animal origin. Virology, 27, 253.

Webster, R.G. \& LAVER, W.G. (1972) The origin of pandemic influenza viruses. In: Influenza Vaccines for Man and Horses. Symposia Series in Immunobiological Standardization, 20, 15. 